\title{
QUEEN'S
UNIVERSITY
BELFAST
}

\section{On the generation of RVE-based models of composites reinforced with long fibres or spherical particles}

Catalanotti, G. (2016). On the generation of RVE-based models of composites reinforced with long fibres or spherical particles. Composite Structures, 138, 84-95. https://doi.org/10.1016/j.compstruct.2015.11.039

Published in:

Composite Structures

Document Version:

Peer reviewed version

Queen's University Belfast - Research Portal:

Link to publication record in Queen's University Belfast Research Portal

Publisher rights

(c) 2016 Elsevier Ltd. This manuscript version is made available under the CC-BY-NC-ND 4.0 license http://creativecommons.org/licenses/by$\mathrm{nc}-\mathrm{nd} / 4.0 /$, which permits distribution and reproduction for non-commercial purposes, provided the author and source are cited.

\section{General rights}

Copyright for the publications made accessible via the Queen's University Belfast Research Portal is retained by the author(s) and / or other copyright owners and it is a condition of accessing these publications that users recognise and abide by the legal requirements associated with these rights.

Take down policy

The Research Portal is Queen's institutional repository that provides access to Queen's research output. Every effort has been made to ensure that content in the Research Portal does not infringe any person's rights, or applicable UK laws. If you discover content in the Research Portal that you believe breaches copyright or violates any law, please contact openaccess@qub.ac.uk. 


\title{
On the generation of RVE-based models of composites reinforced with long fibres or spherical particles
}

\author{
G. Catalanotti ${ }^{\mathrm{a}, \mathrm{b}, *}$ \\ ${ }^{a}$ INEGI, Rua Dr. Roberto Frias, 400, 4200-465 Porto, Portugal \\ ${ }^{\mathrm{b}}$ DEMec, Faculdade de Engenharia, Universidade do Porto, Rua Dr. Roberto \\ Frias, $s / n$, 4200-465 Porto, Portugal
}

\begin{abstract}
An algorithm to create random distributions of uniform spherical or circular particles is proposed here with the aim of generating RVE-based micro-mechanical models for composites reinforced with long fibres or spherical particles. The algorithm is fast, able to represent the randomness of the material and to generate RVEs with any volume fraction, from 0 to its highest theoretical value, i.e. $\frac{\pi}{3 \sqrt{2}} \approx 0.74$ and $\frac{\pi \sqrt{3}}{6} \approx 0.91$ for spheres and circles, respectively. Several statistical spatial descriptors were used to evaluate the goodness of the generated particles' distributions. It was concluded that the proposed algorithm can be used to generate statistically representative distributions of uniform particles.
\end{abstract}

Key words:

Representative Volume Elements (RVEs), Micro-mechanical models, Spatial statistics, Sphere Packing

\section{Introduction}

Understanding the impact of the material micro-structure on the overallstochastic properties represents a major issue in fundamental and applied research on composite materials. Using Representative Volume Elements (RVEs),

\footnotetext{
* Corresponding author

Email address: gcatalanotti@fe.up.pt (G. Catalanotti).
} 
and applying appropriate boundary conditions, micro-mechanical models allow gaining unique insights on the material behaviour that would be impossible, or very difficult, to obtain experimentally. For example, micro-mechanical models could be used to perform multi-functional optimization of composites, to reduce the number of experimental tests required for the material screening and qualification, and to apply stress fields that cannot be applied experimentally (as complex three-dimensional stress states [1]).

Obviously, the material micro-structure plays a central role on the material behaviour. It is therefore necessary to model carefully the micro-structure of the material and to represent with the possible highest level of detail its inherent properties, principally its randomness. Numerous composite materials could be represented as uniform dispersions of circular or spherical particles. Long fibre reinforced composites, for example, could be modelled as uniform dispersions of circular particles (in the transverse cross-section, fibres could be modelled as hard-core circles), while blends of Intrinsic Conductive Polymers (ICPs) with inorganic resins, could be modelled as uniform dispersions of spherical particles.

The random sequential addition (RSA) of hard spheres [2] is usually used when generating micro-mechanical Representative Volume Elements (RVEs) of equal spheres dispersed in a matrix. Its main advantage is the simplicity of the algorithm that generates the random distribution of particles. The algorithm works as follows: i) the coordinates of the centre of the $i$-th particle are randomly generated, ii) it is checked the collision with the other particles already present in the RVE, iii) if there are no collisions the particle is added while if there is collision the particle is not included and the process is iterated.

The main limitation of the RSA is that high volume fractions are virtually impossible to reach. After a critic value of the volume fraction, positioning the next sphere is virtually impossible. The same limitations have other algorithms based on modifications of the RSA.

Circle packing algorithm may be used to create denser packings. However, for circular particle, the jamming limit is 0.547 [3]. Different algorithms were proposed to increase the maximum volume fraction of the RVE. Wongsto and $\mathrm{Li}$ [4] proposed an algorithm based on a modification of a periodic arranged hexagonal lattice but they did not perform the statistical study of the particles' distribution obtained. Additionally, their RVEs were not periodic. This represent a severe limitation and does not allow the use of periodic boundary conditions (PBCs). More recently, Melro et al. proposed an algorithm to generate periodic random distribution of fibres [5] reaching higher volume fractions $\left(v_{f} \approx 0.65\right)$.

For spherical particles, as shown by Jaeger and Nagel [6], random close pack- 
ing of spheres (RCP) in three dimensions gives a packing density of approximately $v_{f} \approx 0.64$ that is smaller than its maximum theoretical value. Later, using molecular dynamic simulations together with the Lubachevsky-Stillinger compression algorithm (LSA)[7], Torquato et al. [8] observed that the problem of random close packing $(\mathrm{RCP})$ is ill-defined and mathematically imprecise. This problem could be overcame introducing the notion of maximally random jammed. The maximum value of packing density obtained is always approximately equal to 0.64 or slightly lower.

However, higher volume fractions may be required in several practical applications. In filament winding, for example, it is possible to obtain, controlling some manufacturing parameters, volume fractions of $v_{f} \approx 0.7$ [9]. Another example is given by textile composites that although having an average volume fraction of $0.5-0.6$ show a very high volume fraction $\left(v_{f} \geq 0.7\right)$ within the yarns [10-13]. No algorithms are available in this case to generate a random distribution of monodispersed particles. The same limitations could arise when modelling uniform dispersions of spherical particles to simulate nanoengineered materials.

Therefore, the main purpose of this work is to present an algorithm for the generation of periodic RVEs of equal hard core particles for any possible volume fraction. It will be shown that the proposed algorithm is fast, able to represent the randomness of the material and that no limitations on the maximum volume fraction exist. The algorithm is, in fact, able to generate random distributions of particles with a volume fraction, $v_{f}$, that ranges from 0 to its highest value equal to $\frac{\pi}{3 \sqrt{2}}$ and $\frac{\pi \sqrt{3}}{6}$ for spheres and circles, respectively $[14,15]$. The details of the algorithm are given in the following for the case of three-dimensional RVEs. The two-dimensional case, being a special case of the three-dimensional one, is reported in Appendix A.

\section{Description of the algorithm}

The main idea put forward here is to generate the random distribution enforcing a perturbation of an ordered disposition of spheres regularly arranged. The RVE is generated in three steps: i) the densest RVE is generated for a given number of spheres (it will be called hereinafter compact $R V E$; ii) the compact $R V E$ is expanded in order to obtain an initial $R V E$ with the desired volume fraction, $v_{f}$, and with spheres periodically arranged and equally distributed; iii) a perturbation (random process) is applied to the initial RVE to obtain the final $R V E$. The algorithm proposed is reported in Figure 1 and it will be described in detail in the following.

[Fig. 1 about here.] 


\subsection{Generation of the compact configuration of the RVE}

It is convenient at this point to start with the densest arrangement of monodisperse spheres. It is well known that this is obtained with two possible dispositions: the face-centred cubic (fcc) and the hexagonal-closed packed (hcp) lattices. Both configurations provide the maximum densest packing but differ in the shape of the unit-cell. The fcc has a cubic cell whilst the hcp has a hexagonal prismatic cell. As in many engineering applications the RVE is chosen to be cubic (for simplicity in the analysis and in the applications of the boundary conditions), the fcc lattice is the natural choice as a starting point. This configuration has to be periodic and it is generated as follows. Consider the spheres of radius $r$ and with centres given by the points:

$$
v_{0}=\left\{\begin{array}{l}
0 \\
0 \\
0
\end{array}\right\}, v_{1}=\left\{\begin{array}{l}
\delta \\
0 \\
\delta
\end{array}\right\}, v_{2}=\left\{\begin{array}{l}
0 \\
\delta \\
\delta
\end{array}\right\}, v_{3}=\left\{\begin{array}{c}
\delta \\
\delta \\
2 \delta
\end{array}\right\}
$$

where $\delta=\sqrt{2} r$. It is easy to show that these can be interpreted as the primitive cell of the lattice (see Figure 2(a)), and that the fcc lattice can be generated translating this cell along the three Cartesian axes (see Figure 2(b)). As the main purpose is to obtain an initial cubic cell with spheres perfectly ordered, this cell is translated of the same amount of times, $M$, along the three different axes. Therefore, the centre of a given sphere in the initial RVE will have coordinates:

$$
v_{i}^{l m n}=v_{i}+\left\{\begin{array}{c}
l-1 \\
m-1 \\
n-1
\end{array}\right\} \delta
$$

for $i=0, . ., 3$ and $l, m, n=1, . ., M$.

If $M$ is the times the cell has been repeated (in all the three directions), the length of the side of the RVE of the lattice, $L_{f c c}$, is given by:

$$
L_{f c c}=M \delta
$$

Notice that the RVE of the lattice is periodic. 


\subsection{Expansion of the compact RVE and generation of the initial configura- tion}

If the actual length of the RVE is $L$, the initial coordinates of the particles may be obtained multiplying them for a correction factor, $f$, given by:

$$
f=L / L_{f c c}
$$

The centres of the spheres in the initial RVE will have coordinates:

$$
\bar{v}_{i}^{l m n}=v_{i}^{l m n} f
$$

The input parameters given to the algorithm are the volume fraction, $v_{f}$, the radius of the particles, $r$, and the minimum length of the RVE, $L_{\text {min }}$. From the radius and the volume fraction, the number of particles, $N_{p}$, is calculated:

$$
N_{p}=4 M^{3}
$$

where $M$ is given by:

$$
M=\left\lceil\left\lfloor\left(\frac{v_{f} L_{\min }^{3}}{4 V_{s}}\right)^{\frac{1}{3}}\right\rceil\right\rceil
$$

and $V_{s}$ is the volume of a particle. $\lceil\cdot\rceil$ and $\lfloor\cdot\rceil$ are the ceiling and round function. The length of the RVE is therefore given by:

$$
L=\left(\frac{V_{s} N_{p}}{v_{f}}\right)^{\frac{1}{3}}
$$

The effective size of the RVE, $L$, will be equal to $L_{\min }$ or slightly larger. Using the ceiling and the round function in equation (7) allows the generation of the RVE with the exact value of volume fraction required, as shown in Figure 2(c).

[Fig. 2 about here.]

\subsection{Perturbation}

The perturbation is given applying a random displacement to all the particles, and this process is iterated $K$ times. It will be shown, in the following, that the 
particles' distribution is completely random for $K>10$, so that the number of iterations can be chosen a priori. Alternatively, $K$ can be a sufficiently large value, and in that case the process could be stopped when a certain criterion is satisfied. To define a proper stopping criterion, one of the spatial descriptor used in the following can be used. For example, one could use the Average Nearest-Neighbour (ANN) distance (see Section 3.3). As explained in the following, this spatial descriptor reaches a plateau when the maximum randomness has been achieved; therefore the loop could be exited when the plateau is reached. It should be noted that, however, the calculation of a statistical spatial descriptor could be time consuming, especially when a large number of particles is considered and therefore, choosing a priori the value of $K$ could substantially speed-up the algorithm.

Every iteration $I$, all the particles are randomly selected and moved. This is achieved within the block $J=\mathcal{R}\left(1, . ., N_{p}\right)$ in the flow chart reported in Figure 1. $\mathcal{R}\left(1, . ., N_{p}\right)$ represents, in fact, a random permutation of the index $\left(1, . ., N_{p}\right)$.

Suppose that $u$ is the random displacement applied to the $i$-th particle. In the particle coordinate system the displacement $u$ can be expressed as

$$
u=\left\{\begin{array}{l}
u_{1} \\
u_{2} \\
u_{3}
\end{array}\right\}=\left\{\begin{array}{c}
\rho \sin \theta \cos \phi \\
\rho \sin \theta \sin \phi \\
\rho \cos \theta
\end{array}\right\}
$$

where $\rho, \theta$, and $\phi$ are the spherical coordinates. To apply a random displacement the polar and azimuthal angles are chosen to be equal to:

$$
\begin{gathered}
\theta=\arccos (2 \mathcal{U}-1) \\
\phi=2 \pi \mathcal{V}
\end{gathered}
$$

where $\mathcal{U}$ and $\mathcal{V}$ are random scalars uniformly distributed on the open interval $(0,1)$. Equations (10) and (11) ensure that all the possible directions are uniformly distributed [16]. The problem of having all the possible directions uniformly distributed is formally equal to that of picking a point from the surface of a sphere. Therefore, the polar and azimuthal angles could also be calculated using different approaches [17-19].

After selecting a random orientation, the maximum radial displacement applicable to the $i$-th particle is computed monitoring the Euclidean distances between the $i$-th particle and the other particles, $d_{i}$. Care should be taken when calculating $d_{i}$, the effective distance between two particles. The RVE is, in fact, periodic and for this reason, if two particles have coordinates $x$ and $y$, 
the effective distance between them is not simply given by

$$
d_{i}=\sqrt{\sum_{j=1}^{3}\left(x_{j}-y_{j}\right)^{2}}
$$

but it should be correctly calculated as:

$$
d_{i}=\sqrt{\sum_{j=1}^{3}\left(\min \left\{\left|x_{j}-y_{j}\right|, L-\left|x_{j}-y_{j}\right|\right\}\right)^{2}}
$$

When $d_{i}=2 r$ the particles come into contact and the maximum radial displacement $\bar{\rho}$ is calculated. The value $\rho$ is obtained as:

$$
\rho=\bar{\rho} \mathcal{W}
$$

where $\mathcal{W}$ is a random scalar uniformly distributed on the open interval $(0,1)$. That allows the calculation of the displacement of equation (9). The new coordinates of the particle reads:

$$
x=x_{0}+u_{i}+\breve{x}
$$

where $x$ are the coordinates after the perturbation and $x_{0}$ the coordinates before the perturbation process. $\breve{x}$ is a correction parameters that takes into account the fact that a given particle cannot abandon the RVE and if a particle exits the RVE in a point, at the same time, the same particle should appear in the symmetric position. This correction parameters reads:

$$
\breve{x}=\left\{\begin{aligned}
0 & \text { if } 0 \leq x_{0}+u_{i} \leq L \\
-L & \text { if } x_{0}+u_{i}>L \\
L & \text { if } x_{0}+u_{i}<0
\end{aligned}\right.
$$

[Fig. 3 about here.]

The following video shows the initialization of the RVE and the generation of the final RVE through all the iterations of the perturbation process.

[Fig. 4 about here.]

[Video 1 about here.]

Finally, Figure 5 shows two RVEs created using the proposed algorithm. The two-dimensional RVE of Figure 5(a) represent the transverse section of a longfibre reinforced composite while the three-dimensional RVE of Figure 5(b) shows a polymer composite with spherical inclusions. Additional details on these models are reported in [20,21].

[Fig. 5 about here.] 


\section{Statistical analysis}

Using the algorithm presented in the previous section, 25 three-dimensional RVEs of monodispersed spherical particles are generated for different volume fractions. Four volume fractions are considered $\left(v_{f}=0.1,0.3,0.5,0.7\right)$ so that representative points in the entire range of the possible values of the volume fraction are considered $\left(0<v_{f} \leq 0.7\right)$. Notice that for these RVEs the highest theoretical value of the volume fraction is equal to $\frac{\pi}{3 \sqrt{2}} \approx 0.74[14,15]$. The side of the RVE is taken approximately 25 times the radius of the particles.

\subsection{Execution time}

The model was implemented in Matlab R2013b [22] and it runs in one cpu of Intel $\cap$ Xeon $\cap$ Processor E5-2650. Figure 6 reports the average of the execution times for the different volume fraction investigated. Error bars represent the standard deviation.

The execution time is reported in function of the perturbation's iterations (see Figure 6). In every iteration all the particles of the RVE are randomly moved. Notice that the algorithm proposed is very fast. In fact, it should be considered that the RVEs are three-dimensional and contains a high number of particles. In the case of the highest volume fraction, $v_{f}=0.7$, the number of particles, $N_{p}$, is equal, for example, to 2916. It should also be taken into account that, as it will be shown in the following, virtual randomness is attained only after 5 iterations. Therefore, regardless of the volume fraction, the execution time necessary to attain a random distribution is less than two minutes for all the RVEs considered. The execution time can also drastically be reduced changing some tolerances in the calculation of the maximum displacement $\bar{\rho}$. In the present work $\bar{\rho}$ is computed numerically with a tolerance lower than $10^{-5} r$, being $r$ the radius of the particle. Relaxing this condition leads to a faster generations of the RVEs.

[Fig. 6 about here.]

\subsection{Volume of Voronoi cells}

A Voronoi diagram (also known as Diriclet tesselation) is defined as the partitioning of a region with $n$ points (generating points) in $n$ sub-regions such that every sub-region contain only one generating point and every point of the sub-region is closer to its generating point than the others [23]. If the considered region is an area, the sub-regions are called Voronoi polygons, whilst if 
the region is a volume, the sub-regions are called Voronoi polyhedra or simply Voronoi cells.

The statistical analysis of the volume of Voronoi cells represents a first important spatial descriptor. In particular, is possible to consider the coefficient of variation of the volume of the Voronoi cells, $c_{v}$ defined as:

$$
c_{v}=\sigma / \mu
$$

where $\mu$ and $\sigma$ are the mean value and the standard deviation respectively. Value of $c_{v}$ equal to zero are obtained for structured arrangements (no randomness) whilst values higher than 0 represent a randomness in the particles' distribution.

Figure 7 reports the coefficients of variation for the different volume fractions considered in this work. The results are reported as error bars, so that each curve represents the mean value of $c_{v}$ (for the 25 RVEs considered and for a given $v_{f}$ ) and the error bar its standard deviations.

[Fig. 7 about here.]

It is shown that the initial value of $c_{v}$ is equal to 0 as expected, being the initial distribution perfectly arranged (fcc lattice). The perturbation promotes an increase of the $c_{v}$ that rapidly attains a constant value. This constant value is reached after few iterations. Denser packings shows lower values of the plateau, as expected, or in other words less randomness in their distribution.

\subsection{Average Nearest-Neighbour distances}

A very useful spatial descriptor is the Average Nearest-Neighbour (ANN) distance $[24,25]$. This is defined as the ratio between the observed mean distance, $\bar{D}_{0}$, and the expected mean distance, $\bar{D}_{E}$, between a particle and its nearest neighbour.

$$
A N N=\bar{D}_{0} / \bar{D}_{E}
$$

For the three-dimensional case [25] the expected value is obtained as

$$
\bar{D}_{E}=\frac{(\Gamma(3 / 2+1))^{\frac{1}{3}} \Gamma(1 / 3+1)}{\pi^{\frac{1}{2}} \lambda^{\frac{1}{3}}} \approx 0.554 \lambda^{-\frac{1}{3}}
$$


where $\Gamma$ is the gamma function and $\lambda$ is the density defined as:

$$
\lambda=N_{p} / L^{3}
$$

$A N N$ is therefore a measure of the dispersion of the particles. For the case of Complete Spatial Randomness (CSR) of infinitesimal points (generated by a homogeneous Poisson process) the value of $A N N$ is equal to 1 . However, if particles of finite radius are considered the theoretical limit of 1 can be reached only for very small values of the volume fraction, $v_{f}[26]$.

In Figure 8 the curves of $A N N$ as a function of the iteration number are reported for different values of the volume fraction.

[Fig. 8 about here.]

Once again the plateau is attained quickly (after only 5-10 iterations). The reaching of the plateau define the attaining of the maximum randomness obtainable with a given geometry and volume fraction, even if a value of 1 is not reached. That is a consequence of the fact that randomness, in the strict sense of the word, cannot exist, owing to the fact that the space occupied by a given particle is unavailable to other particles [26].

\subsection{Nearest neighbour distances}

This spatial descriptor is obtained as the probability density function (p.d.f.) of the distance between one particle and its 1st, 2nd, and 3rd nearest neighbour, and provides information on the short-distance interaction. A careful analysis of the 1st, 2nd, and 3rd p.d.f allows detecting point clusters and regions with local high volume fraction.

Figures 9, 10, 11 show the 1st, 2nd, and 3rd nearest neighbour distance p.d.f, respectively. The p.d.f. are obtained from the RVEs generated using the kernel density estimation (KDE) based on normal distributions.

In Figure 9 the 1st nearest neighbour distance p.d.f is reported. As the interpenetration of the spheres is not physically acceptable, this distribution is defined in the region $\delta_{1} / r \geq 2$. The arrows indicate the initial value of $\delta_{1} / r$ at the beginning of the perturbation (for the fcc lattice).

Observing the figures 9,10, and 11 it can be concluded that the perturbation change the p.d.f. but this change is drastic only during the first ten iterations. After ten iterations the p.d.f. stabilize and further perturbation do not have substantial effects on the particles' distribution. 
The orientations at which the 1st, 2nd, and 3rd nearest particle are positioned can be used to construct another important spatial descriptor. This is now a Cumulative Distribution Function (c.d.f.) that represents the total number of particles that lie along a given direction with respect to a reference particle. For the sake of conciseness only the results on the 1st nearest particle are reported here.

As the problem analysed is three-dimensional, it is possible to define two parameters that provide information on the orientation of the particle (see Section 2.3). The first parameter is given by the azimuth angle $\phi$ (that ranges between zero and $2 \pi$ ); the second parameter will be $\zeta$ defined as:

$$
\zeta=\frac{1}{2}(1+\cos \theta)
$$

when $\zeta$ is a parameter that varies between 0 and 1 (see the analogy with equation (10)). Once again, the kernel density estimation has been used to obtain the c.d.f. of the particles' distribution.

Figure 12 shows the c.d.f. of $\phi$ for different volume fractions. In the case of CSR of infinitesimal points, all the orientations have the same probability, therefore the c.d.f. of the particle orientation is the dashed segment reported in Figure 12. It is possible to notice that the theoretical c.d.f. for perfectly random distributions of particles is somehow approximated by the distributions generated. However, when the volume fractions takes large values, $v_{f} \geq 0.5$, the c.d.f. diverges from the c.d.f. for CSR. This means that not all the directions have the same probability to occur. This should be ascribed to the fact that when large volume fractions are taken into account CSR is lost.

[Fig. 12 about here.]

Analogous conclusions are obtained observing the c.d.f. of $\zeta . \zeta$ varies between 0 and 1 and for CSR the c.d.f. should be a line with slope $1 / 2$. Once again, the perturbation attain a randomness comparable with that of the CSR only for low values of the volume fraction, $v_{f}<0.5$. for high values of $v_{f}$ the c.d.f. 
diverges from that of the CSR and therefore all the orientations do not have the same probability.

[Fig. 13 about here.]

\subsection{Ripley's K-function}

According to Pyrz [27], Ripley's K-function is the most informative spatial descriptor in spatial analysis because is able to provide information about the particles' distribution at several distances from a given particle. $\hat{K}$ is defined as the ratio between the expected number of extra points that lie within a radial distance $h$ from a given distance and the number of points for unit volume [28]. Using the Miles-Lantuéjoul-Stoyan-Hanisch translation edgecorrection $[29,30], \hat{K}$ is often estimated as:

$$
\hat{K}(h)=\frac{V^{2}}{N_{p}^{2}} \sum_{i} \sum_{j \neq i} \frac{\mathcal{I}\left(d_{i j} \leq h\right)}{\gamma_{i j}\left(x_{i}-x_{j}\right)}
$$

where $V$ is the volume of the RVE, $d_{i j}$ is the Euclidean distance between the points $i$ and $j, \mathcal{I}$ is the indicator function, and $\gamma_{i j}$ is the set covariance computed as the volume of the intersection between the RVE and the translated RVE:

$$
\gamma_{i j}\left(x_{i}-x_{j}\right)=\operatorname{vol}\left(B \cap\left(B-\left(x_{i}-x_{j}\right)\right)\right)
$$

being $B$ the region of the RVE, and $\operatorname{vol}(\bullet)$ the volume of $\bullet$.

In this work, being the RVE periodic, it is not necessary to consider the edge correction because for a given searching distance, $h$, it is always possible to count all the points falling into the interesting region. For this purpose, it is necessary to translate the points of the RVE in all the three Cartesian directions. Therefore, the set-covariance always assumes the value $V$ and the Ripley's K-function simply reads:

$$
\hat{K}(h)=\frac{V}{N_{p}^{2}} \sum_{i} \sum_{j \neq i} \mathcal{I}\left(d_{i j} \leq h\right)
$$

To quantify the goodness of the particles' distribution, the Ripley's K-function is compared with that of the Poisson process that reads: 
It is convenient to define a transformation of the equation (24), called Besag's function, that converts the Ripley's K-function for CSR to a horizontal line with equation $\hat{L}=0$ :

$$
\hat{L}(h)=h-\sqrt[3]{\frac{3}{4 \pi} \hat{K}(h)}
$$

Therefore, a statistically representative distribution of particles tends to 0 when increasing $h$; peaks or troughs indicate regularity in the particles' distribution. Figure 14 shows the plots obtained for different volume fractions of the particles. Once again, the perturbation increases the randomness of the particles' distributions. However, this randomness is comparable with that obtained for CSR only for the case of small volume fractions.

[Fig. 14 about here.]

\subsection{Pair correlation function}

Last spatial descriptor used here to evaluate the randomness of the generated distributions of particles is the pair correlation function. This spatial descriptor defines the probability of finding the centre of a particle at a given distance from the centre of another particle and, for short distances, it depends on how the particles are packed together. Formally, it is defined as the probability of finding the centre of a particle inside a spherical shell surrounding a reference particle of internal radius $h$ and thickness $\mathrm{d} h$ :

$$
g(h)=\frac{1}{4 \pi h^{2} N \lambda \mathrm{d} h} \sum_{i=1}^{N} \mathcal{I}\left(\left|d_{i j}-h\right| \leq \frac{\mathrm{d} h}{2}\right) \delta_{i j}
$$

where $\delta_{i j}$ is the Kronecker delta.

The pair correlation function $g(r)$ provides insight on the variation of the density as a function of the distance from a given particle. For structured distributions, $g(r)$ shows sharp peaks corresponding to the distance between the arranged particles. For the case of CSR, $g(r)=1$. Therefore, a random distribution of particles should be characterized by $g(r)$ tending to 1 when the distance $h$ increases. 
Figure 15 shows the pair correlation function for the volume fractions investigated. It can be concluded that the perturbation really increase the randomness of the particles' distribution. However, for very large values of volume fraction, even if $g(r)$ tends to $1, g(r)$ shows peaks and troughs and this indicates that the distribution is random but not comparable to the case of CSR.

[Fig. 15 about here.]

\section{Conclusions}

In this paper, an algorithm was proposed to generate random distributions of mono-dispersed spherical and circular particles. The algorithm is fast, able to represent the randomness of the material and to generate RVEs with any volume fraction, from 0 to its highest value equal to $\frac{\pi}{3 \sqrt{2}}$ and $\frac{\pi \sqrt{3}}{6}$ for spheres and circles, respectively.

It should be noticed that the proposed algorithm is able to generate RVEs with the exact value of the desired volume fraction. This fact is very relevant when the RVE is used to estimate an overall property of interest. Being this property stochastic (because the particles' distribution is random), a statistical analysis is usually performed to study its statistical distribution. Eliminating the uncertainty on the volume fraction simplify enormously this task.

Several statistical spatial descriptors were used to assess the quality of the particles' distribution obtained. The results confirm that the proposed algorithm allows the generation of random distribution of monodispersed particles in a very efficient way (the maximum randomness is reached quickly, only after 5-10 iterations). The maximum degree of randomness reached is comparable to the case of CSR only for very small volume fractions. For large values of the volume fraction, randomness is lost and particles own a certain structures. This does not depend on the algorithm but on the fact that particles have a finite dimension.

The RVEs generated will be used to evaluate the impact of the particles' distribution on the overall stochastic properties of composite materials.

\section{Acknowledgments}

The author would like to thank project NORTE-07-024-FEDER-000033 Composite Materials, Structures and Processes, within the Portuguese Na- 
tional Strategic Reference Framework (QREN), through the European Regional Development Fund (ERDF).

\section{Appendix A: Generation of two-dimensional RVEs}

The algorithm described in figure 1 can easily be modified to generate uniform dispersions of hard circular particles, as those proposed previously by other authors $[4,5]$. The generation of two-dimensional RVEs can obtained as a special case of the algorithm used to generate three-dimensional RVEs. The compact RVE is generated from a hexagonal pattern as shown in figure 16. As previously done, the compact RVE is generated translating the unit cell. The circles of the unit cell have coordinates:

$$
v_{0}=\left\{\begin{array}{l}
0 \\
0
\end{array}\right\}, v_{1}=\left\{\begin{array}{c}
r \\
\sqrt{3} r
\end{array}\right\}
$$

while a given circle in the initial RVE will have coordinates:

$$
v_{i}^{m n}=v_{i}+\left\{\begin{array}{c}
(m-1) \delta_{x} \\
(n-1) \delta_{y}
\end{array}\right\}
$$

where $\delta_{x}=2 r, \delta_{y}=2 \sqrt{3} r, i=0,1, m=1, . ., M, n=1, . ., N$, being $N$ and $M$ the times that the unit cell is repeated along $x$ and $y$ respectively.

[Fig. 16 about here.]

The initial RVE, showed in figure 17 is obtained as described in the previously section.

[Fig. 17 about here.]

Finally, as the two-dimensional algorithm is a special case of the threedimensional algorithm, only two parameters plays a role during the perturbation, namely $\rho$ and $\phi$. Those are calculated as already reported in Equations (14) and (11). The final RVE, after the perturbation, is reported in figure 18.

[Fig. 18 about here.] 


\section{References}

[1] P.P. Camanho, A. Arteiro, A.R. Melro, G. Catalanotti, and M. Vogler. Threedimensional invariant-based failure criteria for fibre-reinforced composites. International Journal of Solids and Structures, 55(0):92 - 107, 2015.

[2] B. Widom. Random sequential addition of hard spheres to a volume. J. Chem. Phys., 44:3888, 1966.

[3] Jens Feder. Random sequential adsorption. Journal of Theoretical Biology, 87(2):237 - 254, 1980.

[4] A. Wongsto and S. Li. Micromechanical $\{\mathrm{FE}\}$ analysis of $\{\mathrm{UD}\}$ fibre-reinforced composites with fibres distributed at random over the transverse cross-section. Composites Part A: Applied Science and Manufacturing, 36(9):1246 - 1266, 2005.

[5] A.R. Melro, P.P. Camanho, and S.T. Pinho. Generation of random distribution of fibres in long-fibre reinforced composites. Composites Science and Technology, 68(9):2092 - 2102, 2008.

[6] H.M. Jaeger and S.R. Nagel. Physics of granular states. Science, 255:1523-1531, 1992.

[7] Boris D. Lubachevsky and Frank H. Stillinger. Geometric properties of random disk packings. Journal of Statistical Physics, 60(5-6):561-583, 1990.

[8] S. Torquato, T. M. Truskett, and P. G. Debenedetti. Is random close packing of spheres well defined? Phys. Rev. Lett., 84:2064-2067, Mar 2000.

[9] R. Gibson. Principles of composite material mechanics. Boca Raton, FL: CRC Press., second edition, 2007.

[10] Brian N. Cox and Gerry Flanagan. Handbook of analytical methods for textile composites. Technical Report NASA Contractor Report 4750, NASA - National Aeronautics and Space Administration, Langley Research Center Hampton, Virginia 23681-0001, March 1997.

[11] P. Potluri, I. Parlak, R. Ramgulam, and T.V. Sagar. Analysis of tow deformations in textile preforms subjected to forming forces. Composites Science and Technology, 66(2):297 - 305, 2006. Experimental Techniques and Design in Composite Materials Experimental Techniques and Design in Composite Materials.

[12] J. Xu, S.V. Lomov, I. Verpoest, S. Daggumati, W. Van Paepegem, and J. Degrieck. A progressive damage model of textile composites on mesoscale using finite element method: Fatigue damage analysis. "Computers 83 Structures", 152(0):96-112, 2015.

[13] Mark Pankow, Anthony M. Waas, Chian-Fong Yen, and Seth Ghiorse. A new lamination theory for layered textile composites that account for manufacturing 
induced effects. Composites Part A: Applied Science and Manufacturing, 40(12):1991-2003, 2009. Special Issue: CompTest 2008.

[14] C.F. Gauss. Besprechung des Buchs von L.A. Seeber: Intersuchungen über die Eigenschaften der positiven ternären quadratischen Formen usw. Göttingsche Gelehrte Anzeigen., 1831. In German.

[15] John Conway and Neil J. A. Sloane. Sphere Packings, Lattices and Groups. Springer-Verlag New York, Inc., 1998.

[16] Eric W. Weisstein. Sphere point picking. From MathWorld-A Wolfram Web Resource. http://mathworld.wolfram.com/SpherePointPicking.html.

[17] J von Neumann. Various techniques used in connection with random digits. NBS Appl. Math. Ser., 12:36-38, 1951. Washington, DC: U.S. Government Printing Office.

[18] J. M. Cook. Technical notes and short papers: Rational formulae for the production of a spherically symmetric probability distribution. Math. Tables Aids Comput., 11:81-82, 1957.

[19] G. Marsaglia. Choosing a point from the surface of a sphere. Ann. Math. Stat., 43:645-646, 1972.

[20] G. Catalanotti, M. Bodaghi, and N. Correia. On the statistics of transverse permeability of randomly distributed fibres. Composites A. under review.

[21] G. Catalanotti and A. Katunin. Modelling the electro-mechanical properties of Ppy/epoxy conductive composites. Computational Material Science. Accepted for publication.

[22] The MathWorks, Inc., Natick, Massachusetts, United States. MATLAB R2013b.

[23] A. Bowyer. Computing dirichlet tessellations. Comput J, 24(2):162-6, 1981.

[24] Philip J. Clark and Francis C. Evans. Distance to Nearest Neighbor as a Measure of Spatial Relationships in Populations. Ecology, 35(4):445-53, 1954.

[25] Philip J. Clark and Francis C. Evans. Generalization of a Nearest Neighbor Measure of Dispersion for Use in K Dimensions. Ecology, 60(2):316-17, 1979.

[26] P.P. Bansal and A.J. Ardell. Average Nearest-Neighbor Distances Between Uniformly Distributed Finite Particles. Metallography, 5:97-111, 1972.

[27] Ryszard Pyrz. Quantitative description of the microstructure of composites. part i: Morphology of unidirectional composite systems. Composites Science and Technology, 50(2):197 - 208, 1994.

[28] Philip M. Dixon. Ripley's k function. In Abdel H. El-Shaarawi and Walter W. Piegorsch, editors, Encyclopedia of Environmetrics, pages 1796-1803. John Wiley \& Sons, Ltd., 2002.

[29] J. Ohser. On estimators for the reduced second moment measure of point processes. Series Statistics, 14(1):63-71, 1983. 
[30] A. J. Baddeley, R. A. Moyeed, C. V. Howard, and A. Boyde. Analysis of a threedimensional point pattern with replication. Journal of the Royal Statistical Society. Series C (Applied Statistics), 42(4):641-668, 1993. 


\section{List of Figures}

1 Flow-chart of the algorithm. 20

2 Initialization of the RVE. 21

3 Perturbation. 22

4 Generation of the final RVE (refer to the supplementary material uploaded).

5 RVE-based Finite Element models generated with the proposed algorithm.

6 Cumulative time. 25

$7 \quad$ Mean and standard deviation voronoi cells volume. 26

$8 \quad$ Average Nearest-Neighbour distance. 27

$9 \quad$ 1st Nearest neighbour distances. 28

10 2nd Nearest neighbour distances. 29

11 3rd Nearest neighbour distances. 30

12 Cumulative Distribution Function of $\phi$.

13 Cumulative Distribution Function of $\zeta$. 32

14 Besag's function. 33

15 Pair correlation function. 34

16 Compact RVE (2D). 35

17 Initial RVE (2D). 36

18 Final RVE (2D). $\quad 37$ 


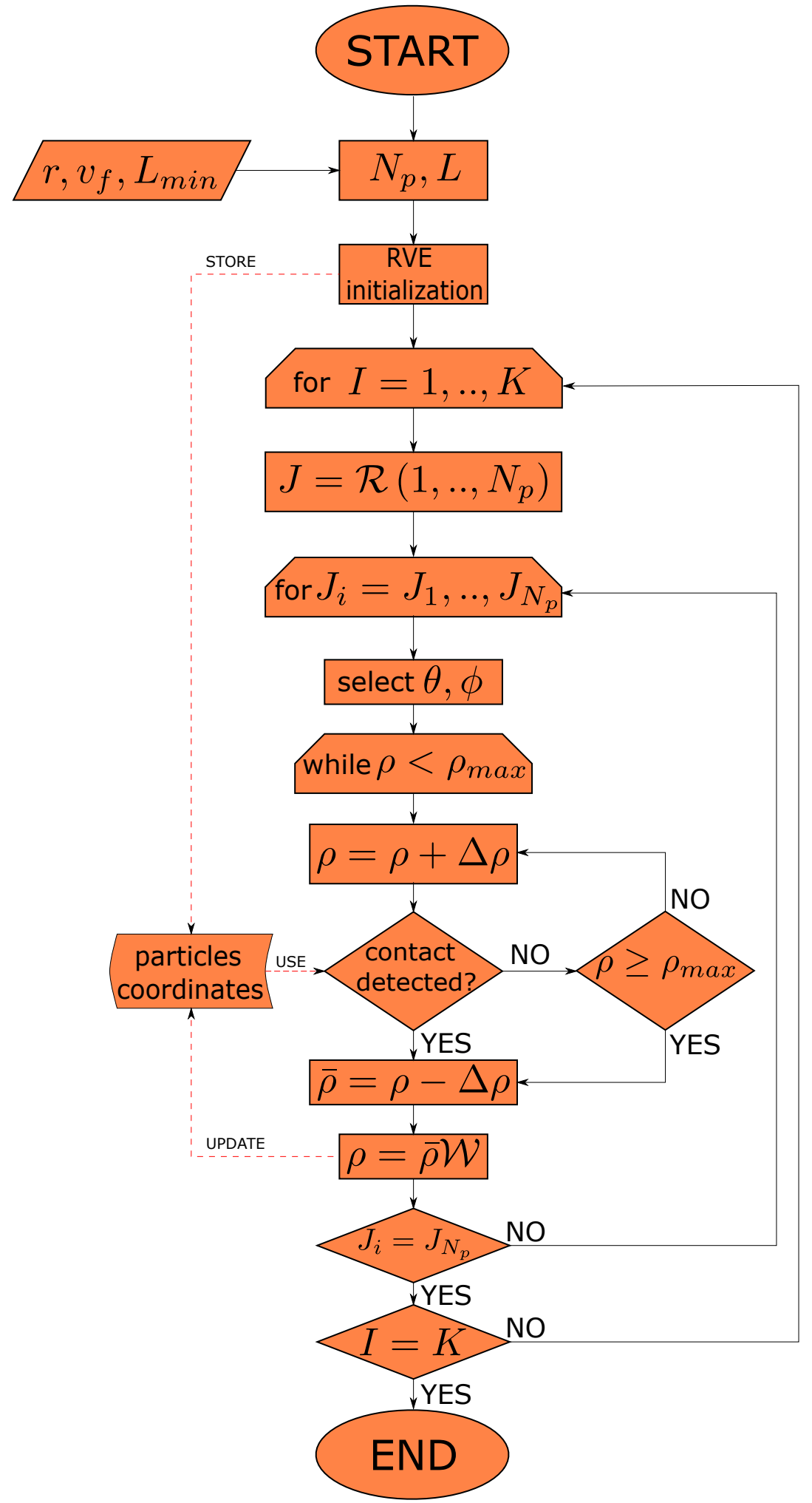

Fig. 1. Flow-chart of the algorithm. 


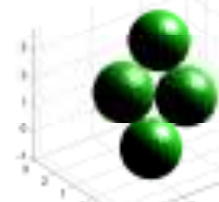

(a) primitive cell

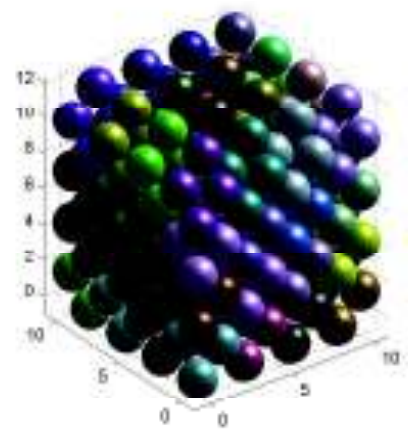

(b) compact RVE

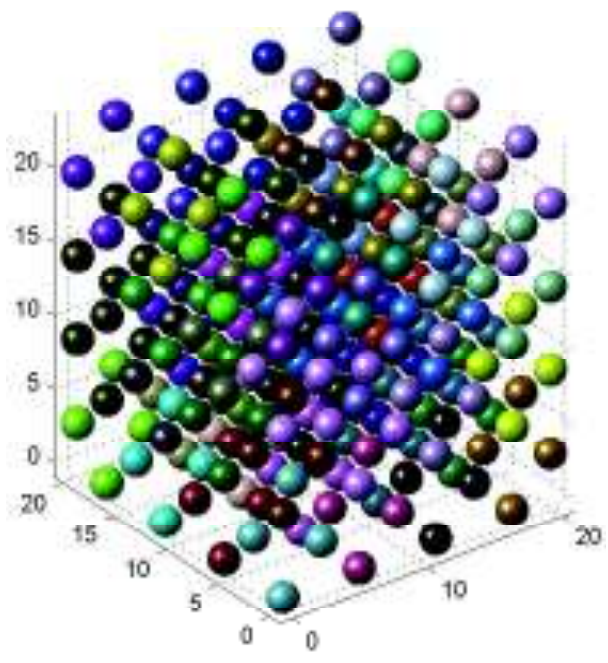

(c) initial RVE

Fig. 2. Initialization of the RVE. 


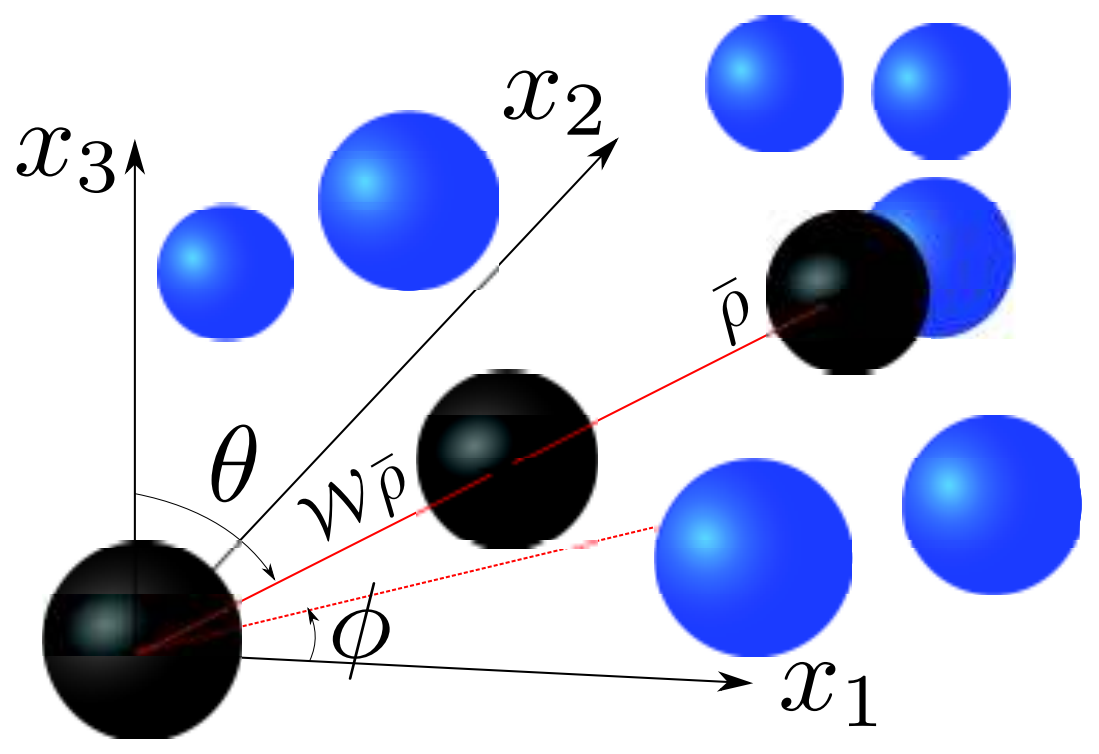

Fig. 3. Perturbation. 


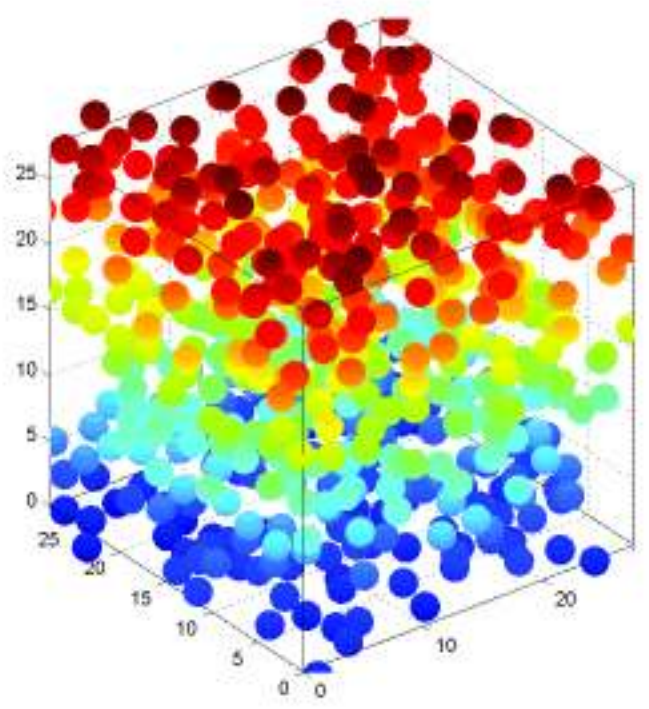

Fig. 4. Generation of the final RVE (refer to the supplementary material uploaded). 


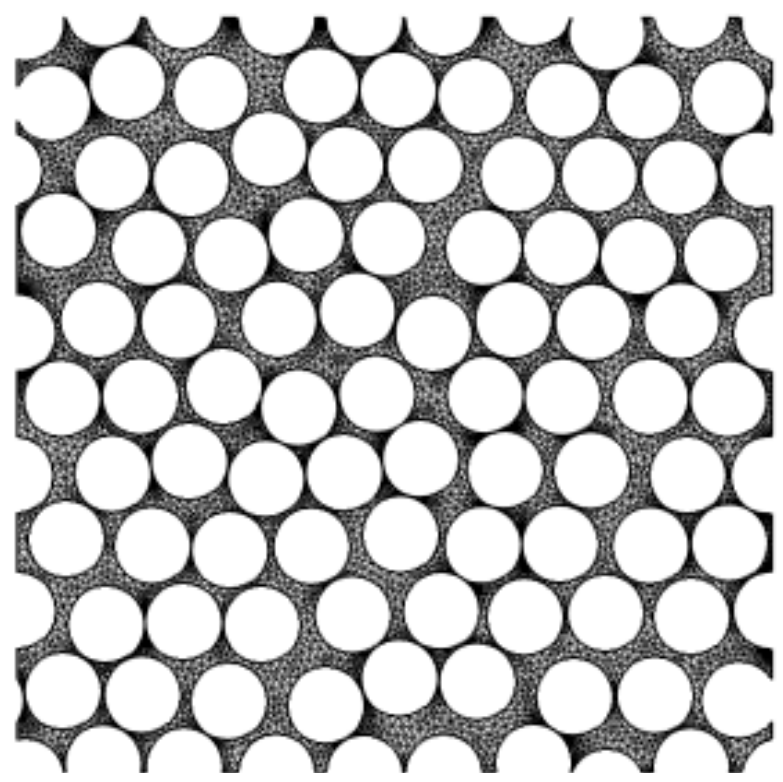

(a) Two-dimensional RVE.

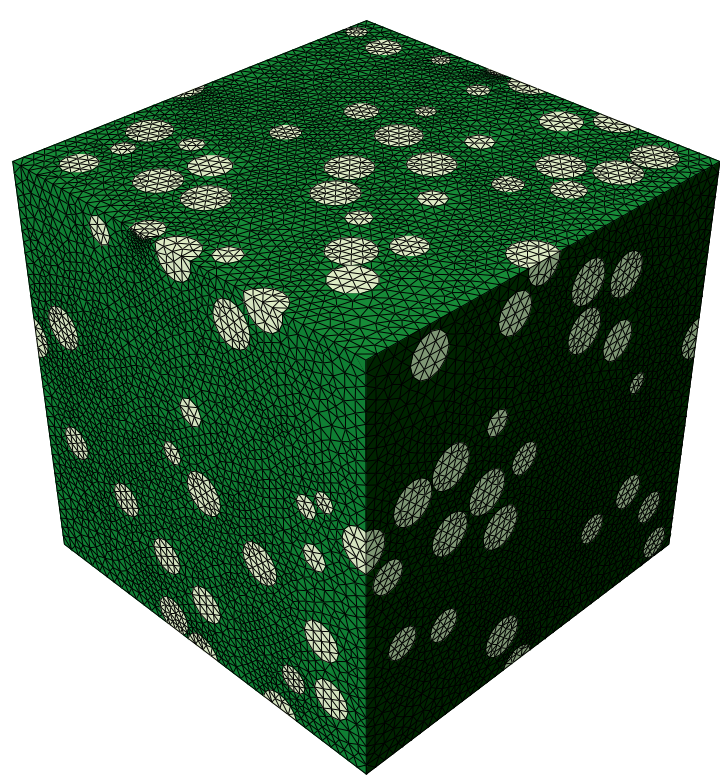

(b) Three-dimensional RVE.

Fig. 5. RVE-based Finite Element models generated with the proposed algorithm. 


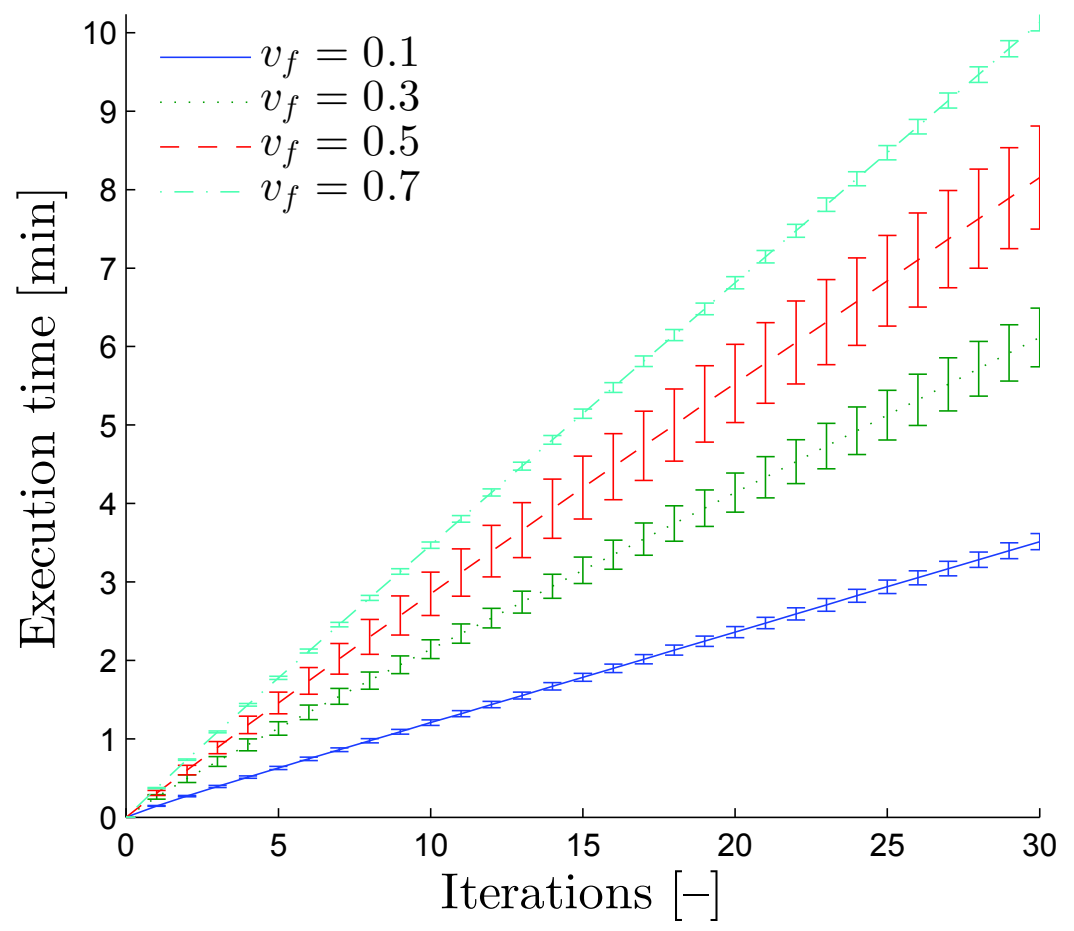

Fig. 6. Cumulative time. 


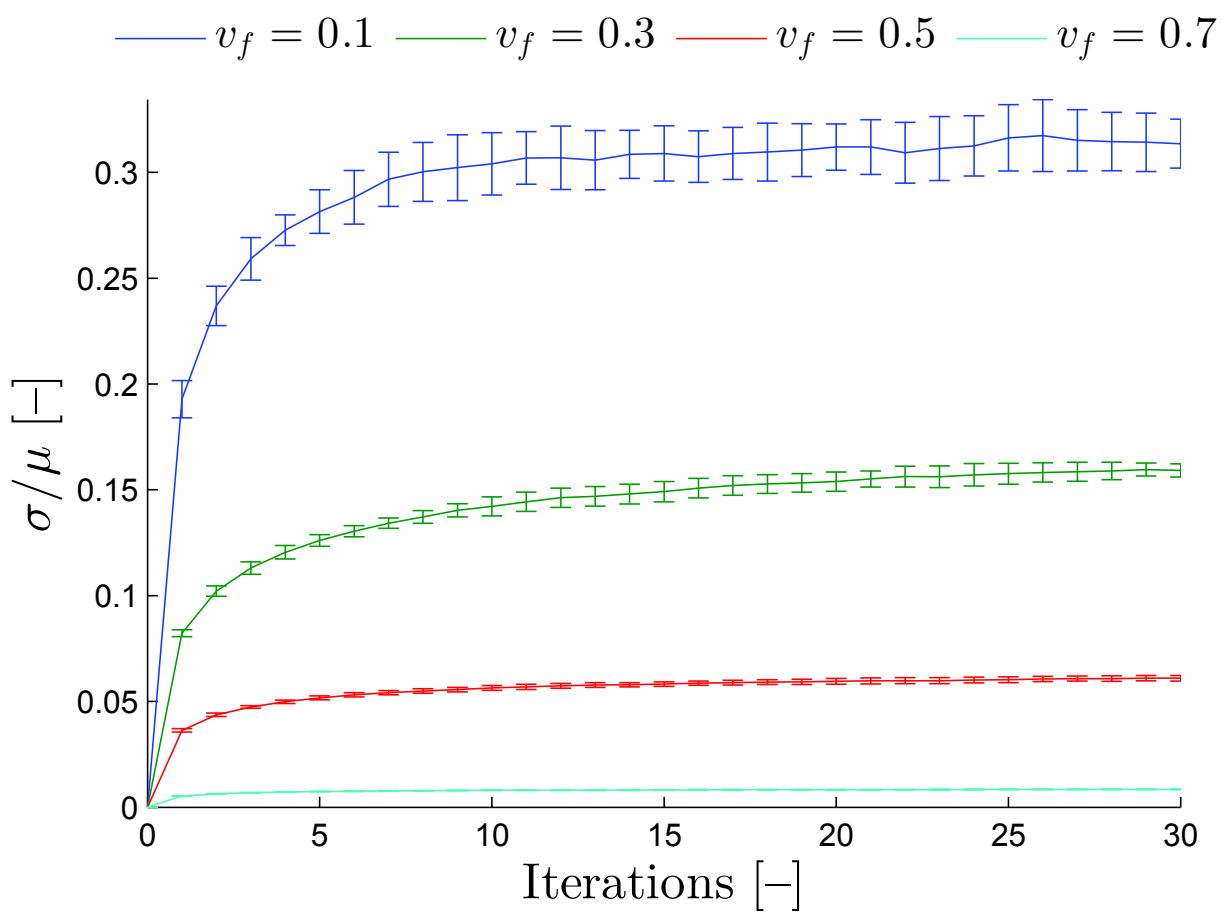

Fig. 7. Mean and standard deviation voronoi cells volume. 


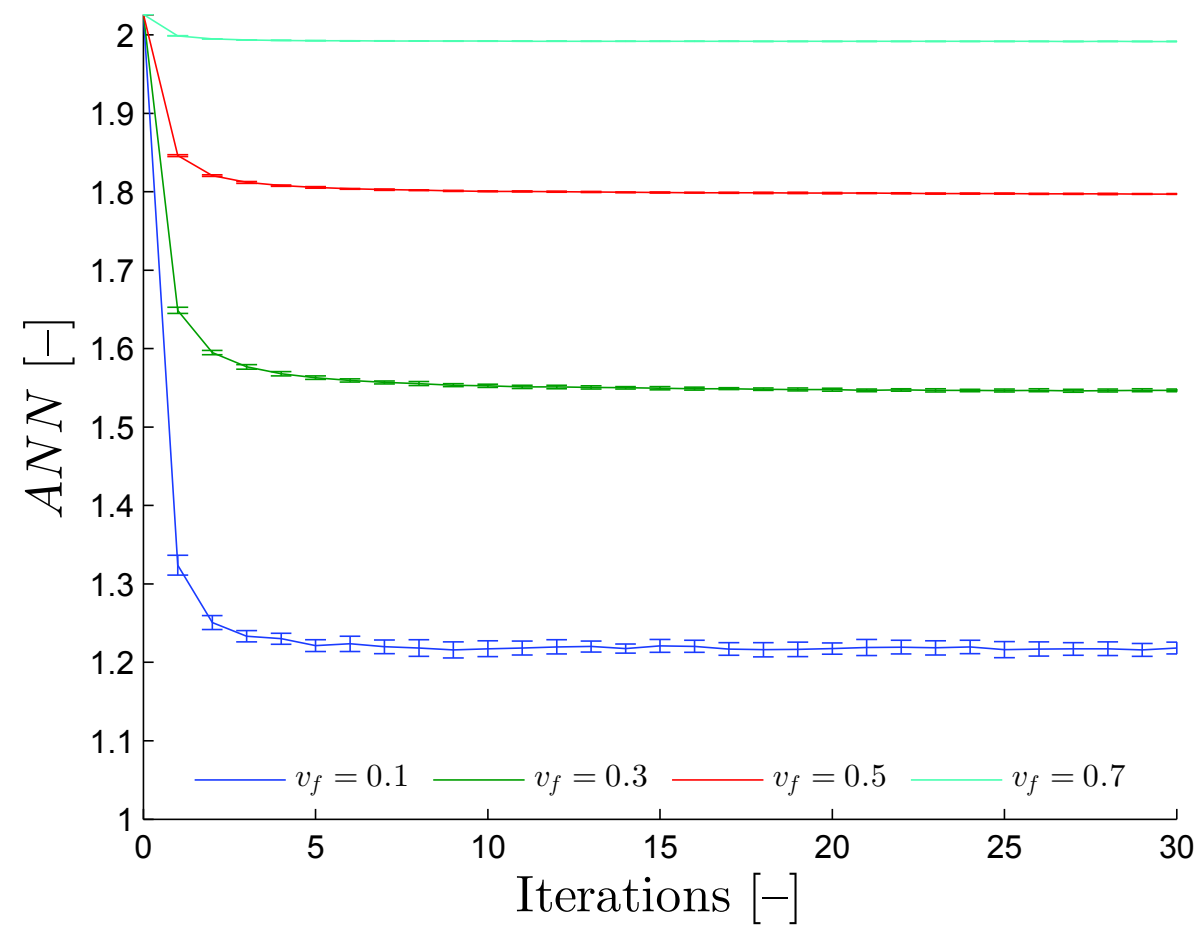

Fig. 8. Average Nearest-Neighbour distance. 


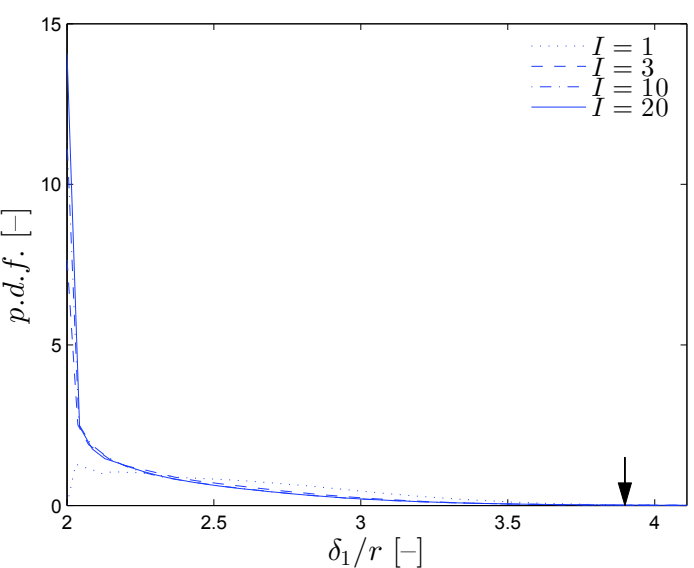

(a) $v_{f}=0.1$

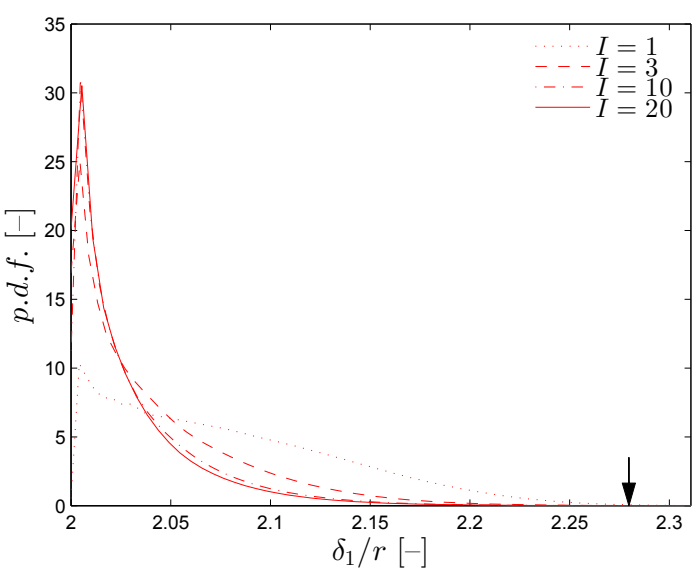

(c) $v_{f}=0.5$

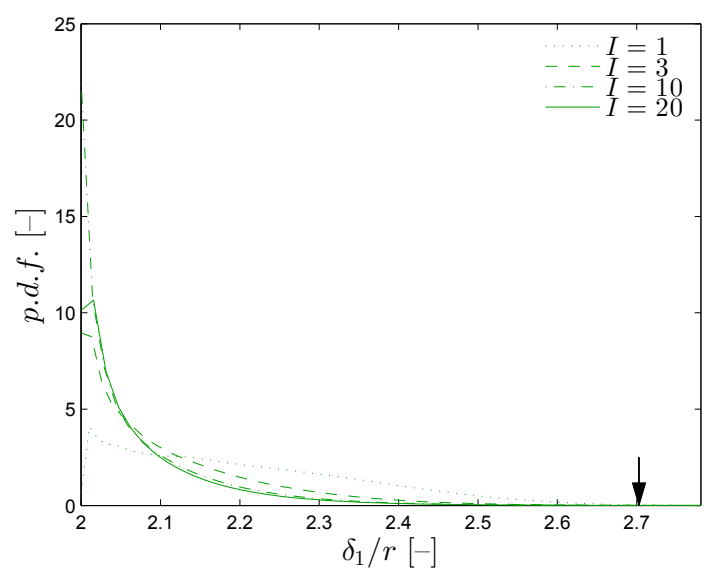

(b) $v_{f}=0.3$

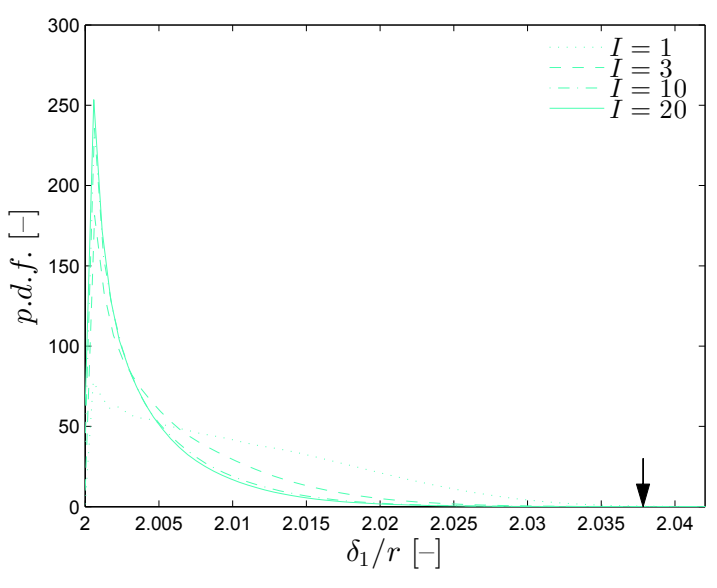

(d) $v_{f}=0.7$

Fig. 9. 1st Nearest neighbour distances. 


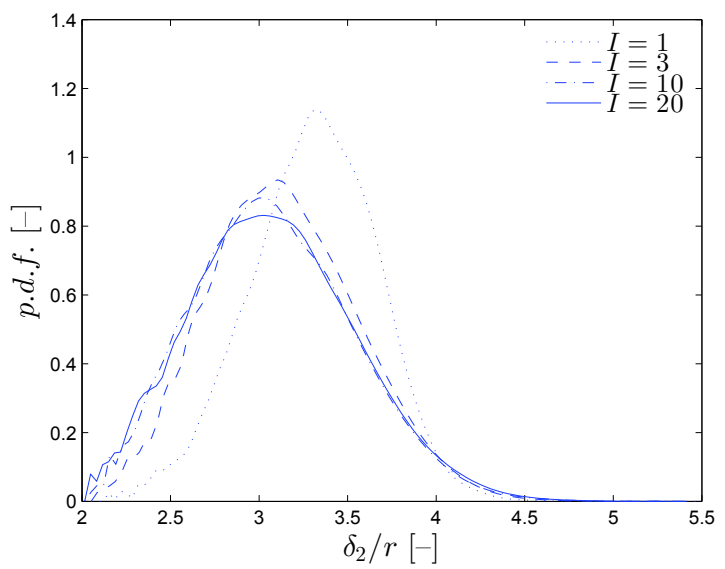

(a) $v_{f}=0.1$

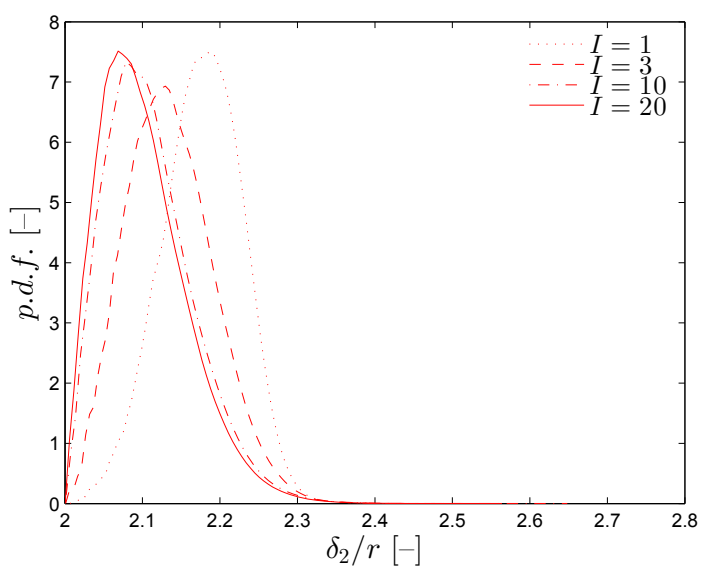

(c) $v_{f}=0.5$

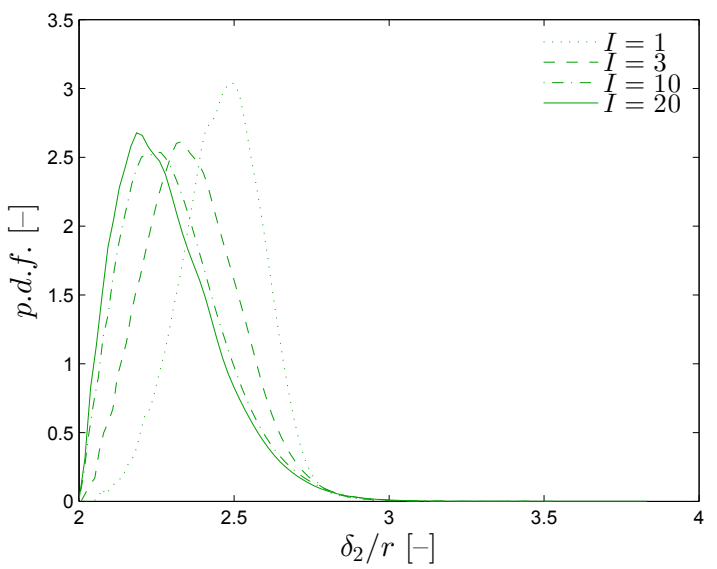

(b) $v_{f}=0.3$

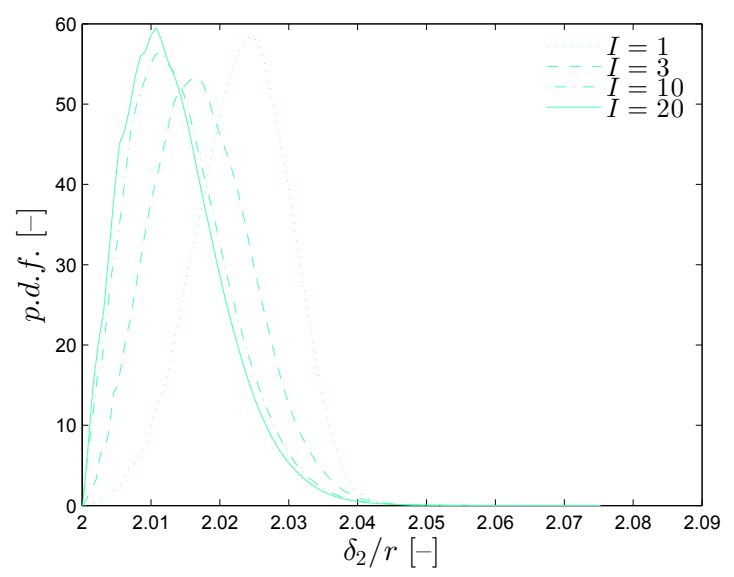

(d) $v_{f}=0.7$

Fig. 10. 2nd Nearest neighbour distances. 


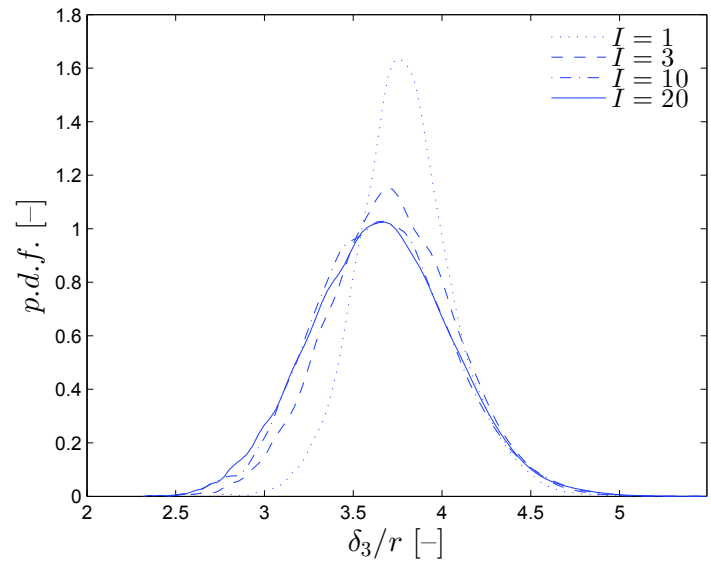

(a) $v_{f}=0.1$

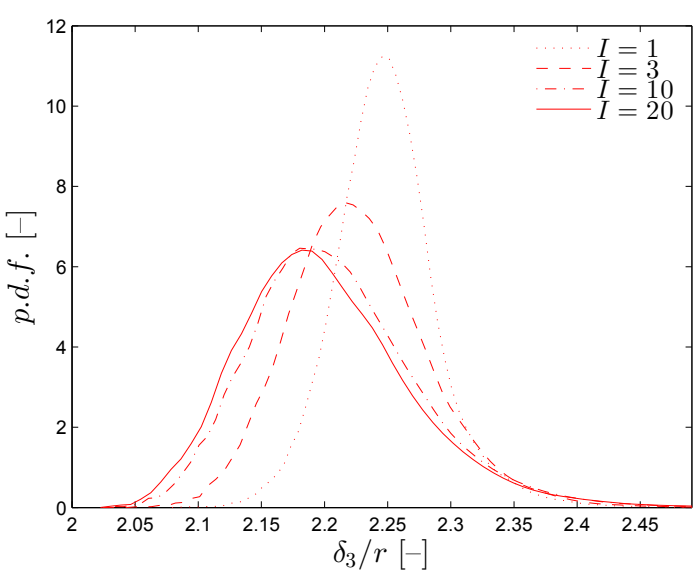

(c) $v_{f}=0.5$

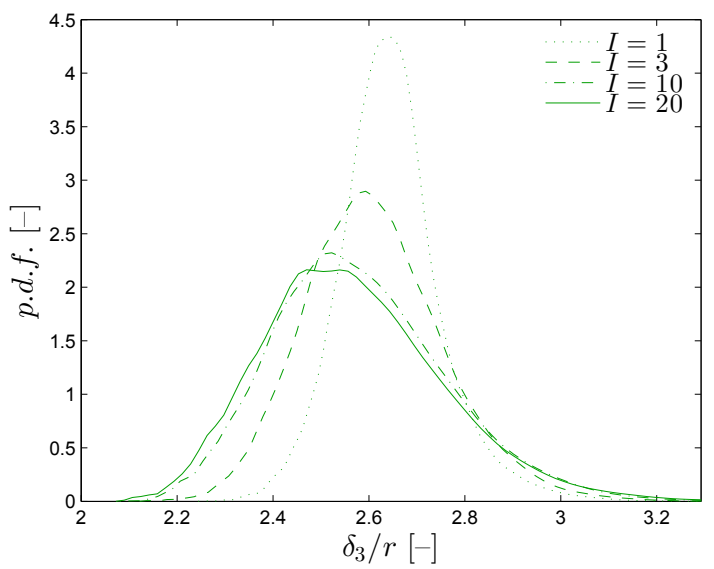

(b) $v_{f}=0.3$

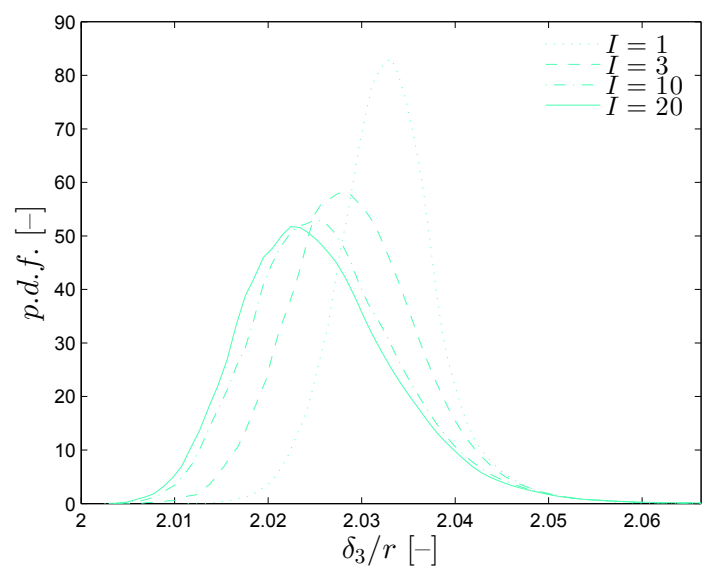

(d) $v_{f}=0.7$

Fig. 11. 3rd Nearest neighbour distances. 


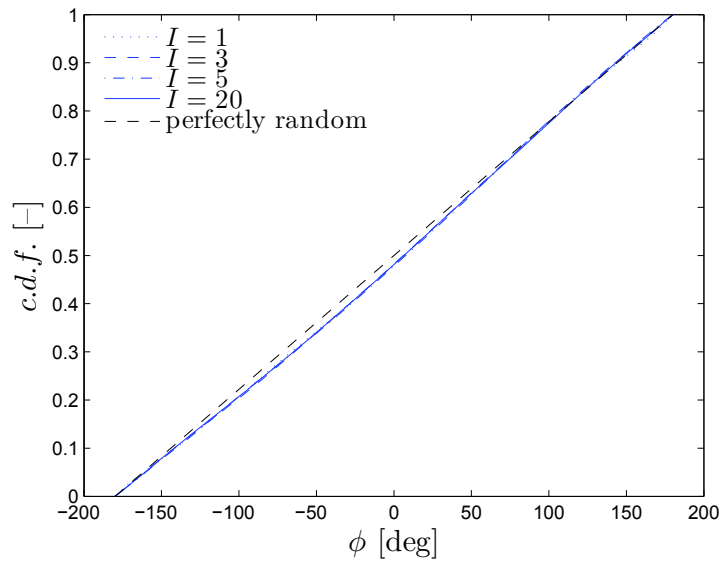

(a) $v_{f}=0.1$

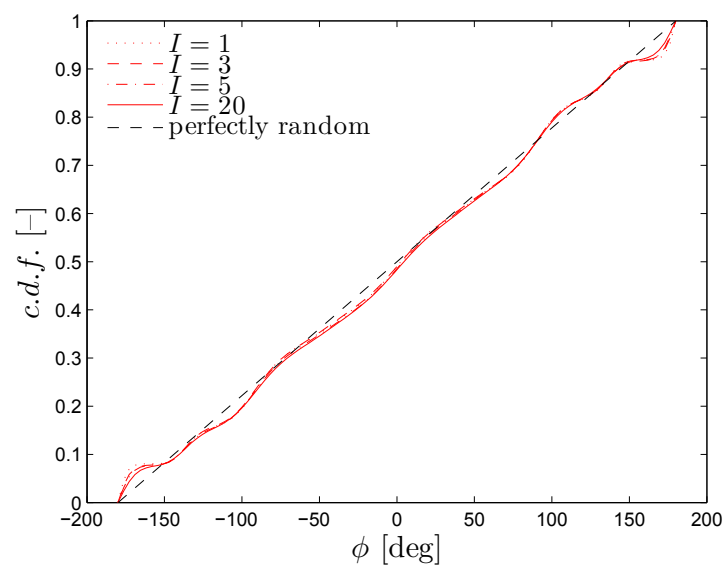

(c) $v_{f}=0.5$

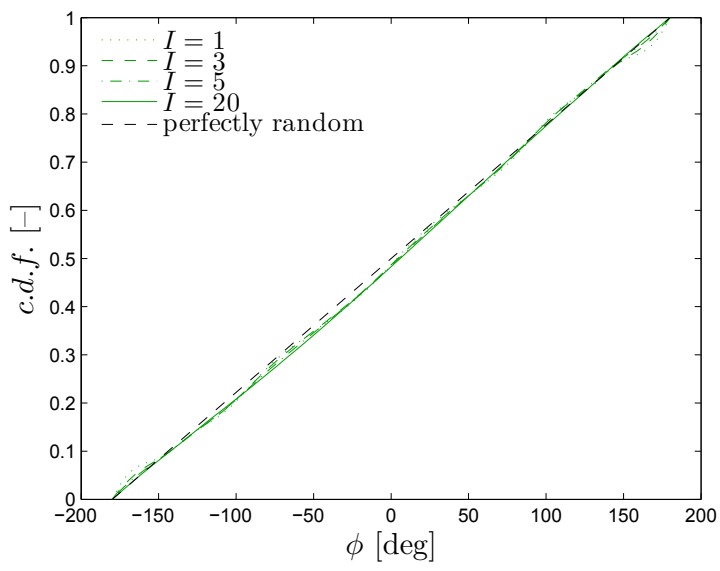

(b) $v_{f}=0.3$

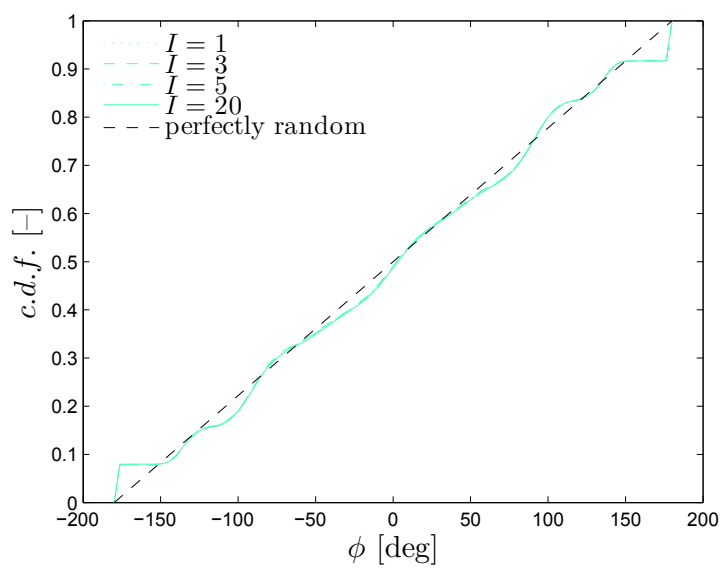

(d) $v_{f}=0.7$

Fig. 12. Cumulative Distribution Function of $\phi$. 


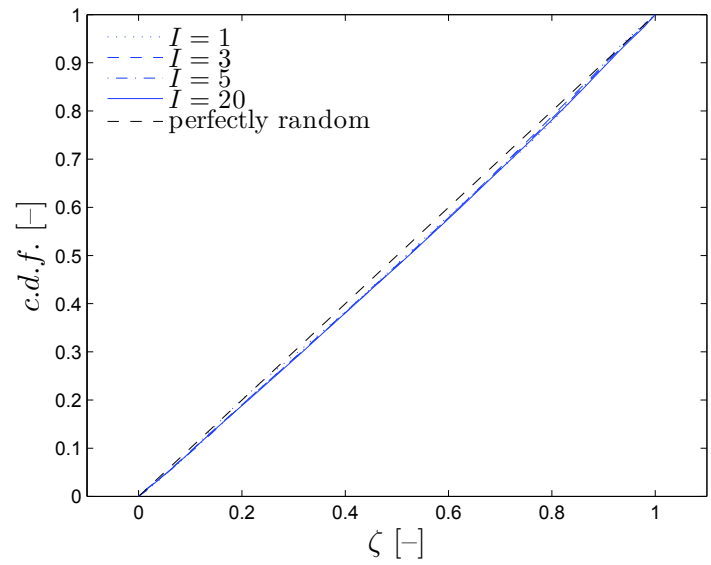

(a) $v_{f}=0.1$

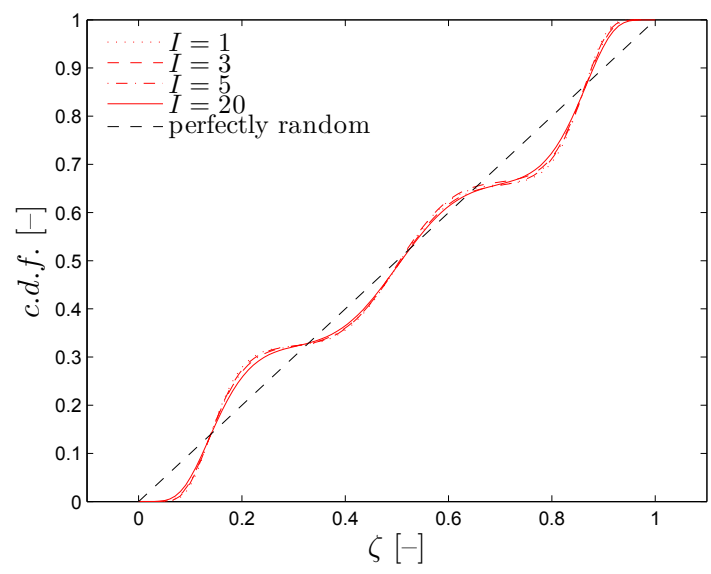

(c) $v_{f}=0.5$

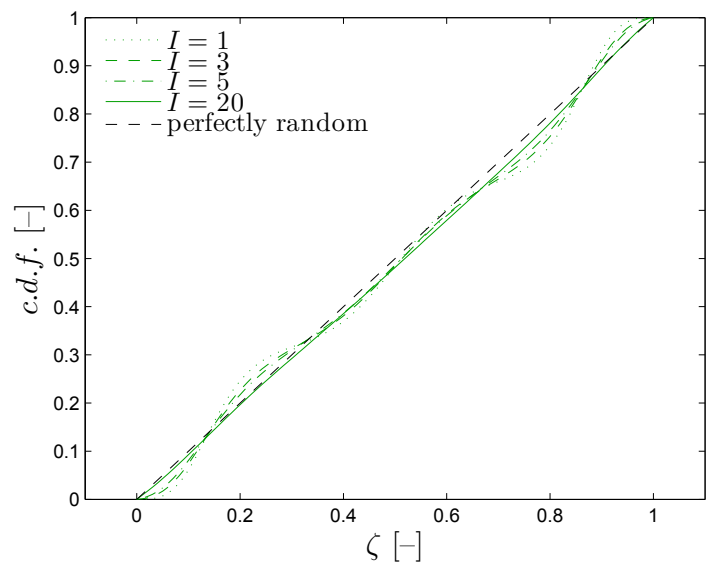

(b) $v_{f}=0.3$

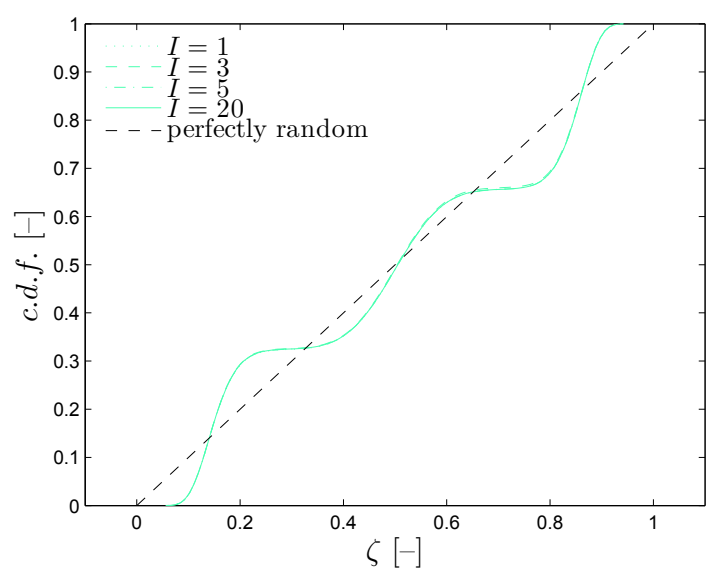

(d) $v_{f}=0.7$

Fig. 13. Cumulative Distribution Function of $\zeta$. 


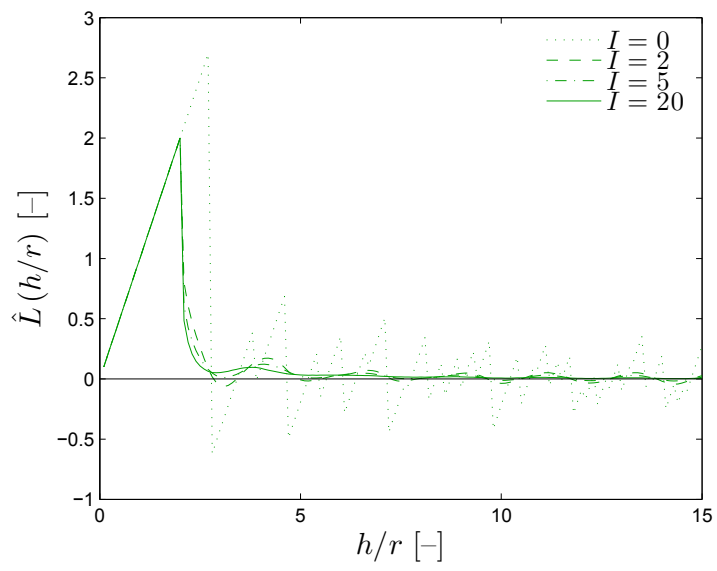

(b) $v_{f}=0.3$

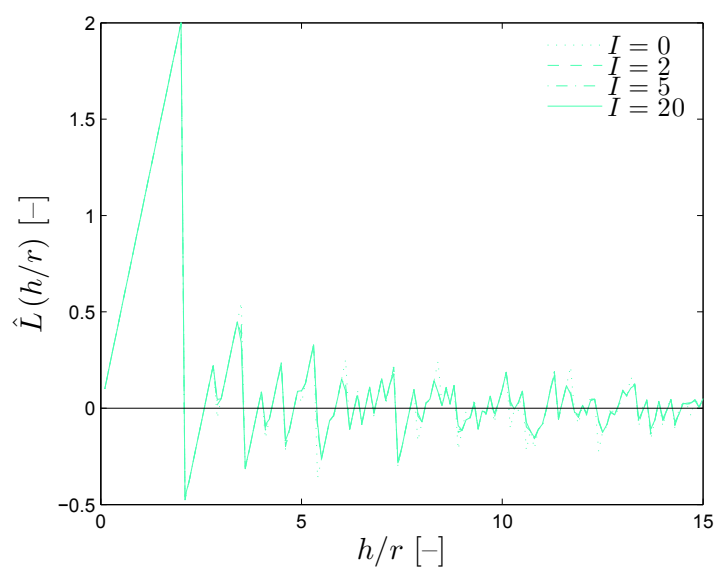

(d) $v_{f}=0.7$

Fig. 14. Besag's function. 


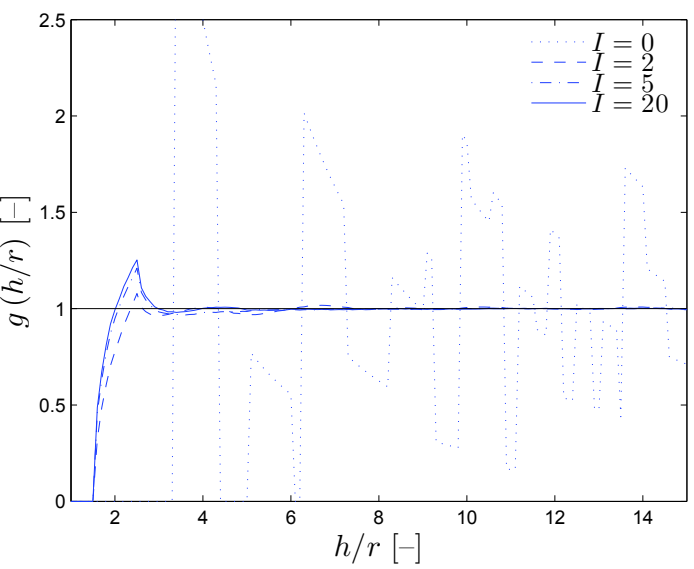

(a) $v_{f}=0.1$

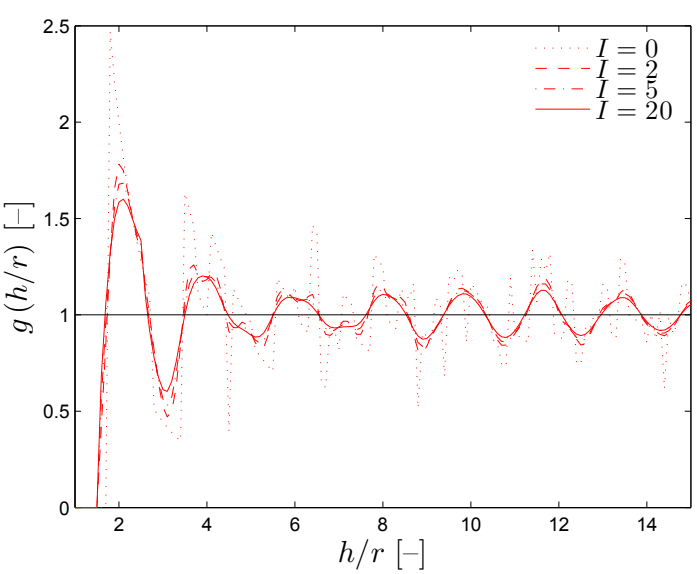

(c) $v_{f}=0.5$

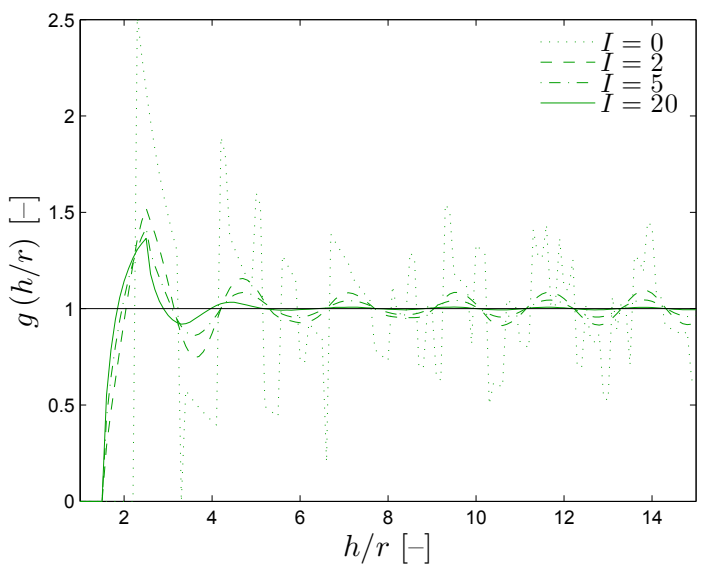

(b) $v_{f}=0.3$

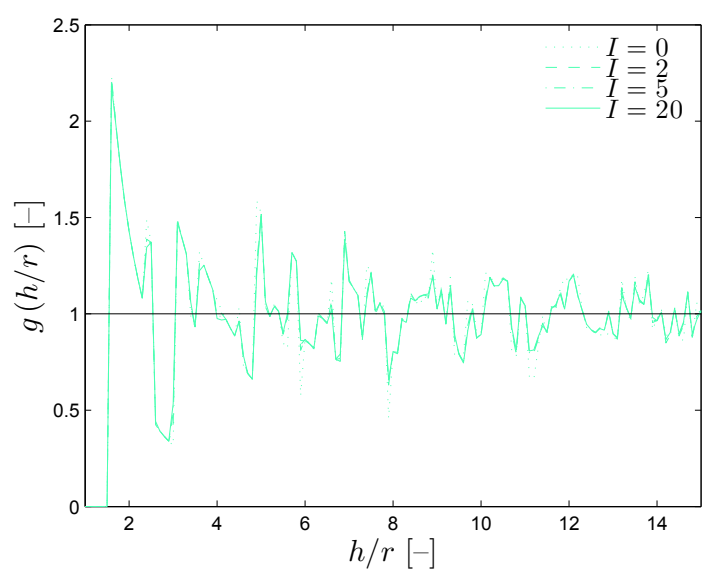

(d) $v_{f}=0.7$

Fig. 15. Pair correlation function. 


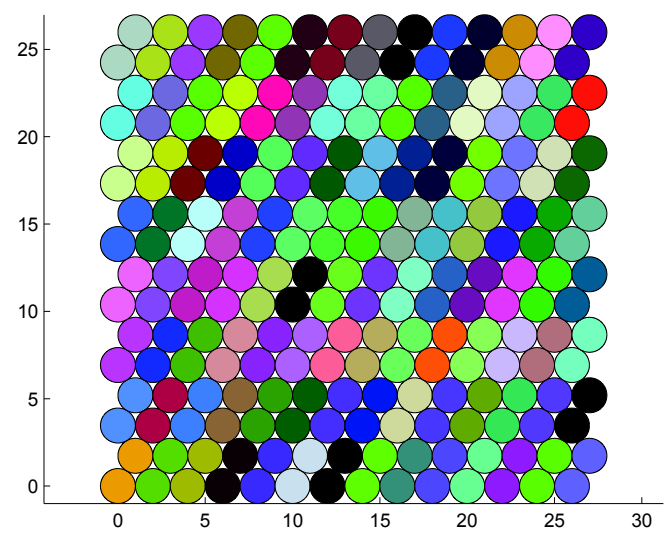

Fig. 16. Compact RVE (2D). 


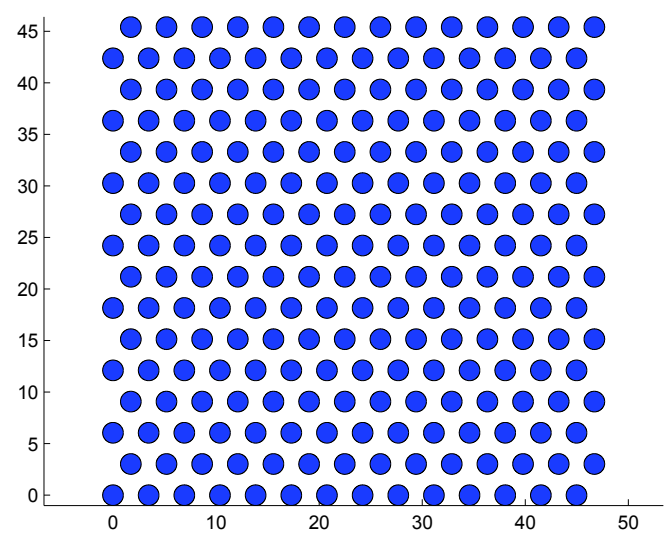

Fig. 17. Initial RVE (2D). 


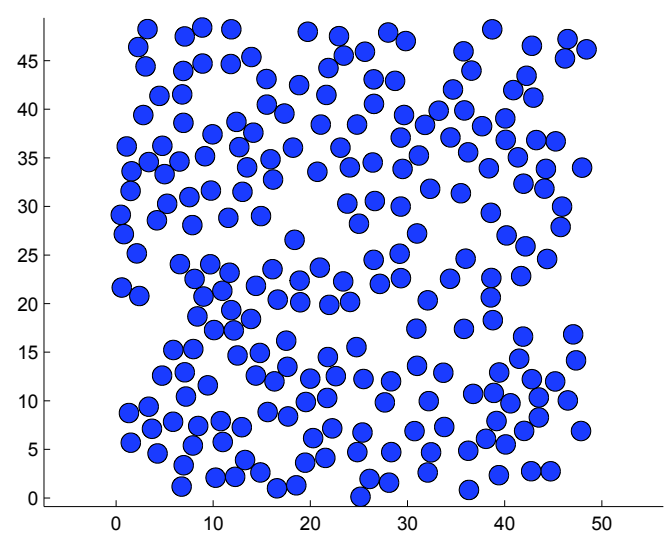

Fig. 18. Final RVE (2D). 